\title{
P I 6-47. Interleukin (IL)-2 I induces cytolytic molecule perforin in CD4 and CD8 T cells without CD4 activation in chronically SIV infected rhesus macaques
}

\author{
S Pahwa*1, S Pallikkuth ${ }^{1}$, KA Rogers ${ }^{2}$, M Doster $^{3}$, F Villinger ${ }^{2}$ and \\ G Franchini ${ }^{3}$
}

Address: ${ }^{1}$ University of Miami Miller School of Medicine, Miami, FL, USA, ${ }^{2}$ Emory University, Atlanta, GA, USA and ${ }^{3}$ National Cancer Institute, Bethesda, MD, USA

* Corresponding author

from AIDS Vaccine 2009

Paris, France. 19-22 October 2009

Published: 22 October 2009

Retrovirology 2009, 6(Suppl 3):P276 doi:10.1 I86/I742-4690-6-S3-P276

This abstract is available from: http://www.retrovirology.com/content/6/S3/P276

(C) 2009 Pahwa et al; licensee BioMed Central Ltd.

\section{Background}

IL-21 is a common g-chain utilizing cytokine derived mainly from activated CD4 T cells and T follicular helper cells with a broad range of cellular targets. We previously described perforin induction by IL-21 in human CD8 T cells with dependence on prior cellular activation and Stat-3 utilization (Blood, 2007).

\section{Methods}

To confirm its biologic activity, we conducted pilot in vivo studies of r-Mamu IL-21 in rhesus macaques.

\section{Results}

In two healthy animals a dose escalation study (1, 10, 50 and 100 microgram/kg subcutaneous IL-21 at 14 day intervals) resulted in a modest transient increase in perforin in T cells. There were no adverse events but anti-IL21 antibody mainly to his-tag was rapidly induced. In 4 chronically SIV infected animals, IL-21 injections (2 doses, 50 microgram $/ \mathrm{kg}$ intravenously at 7 day intervals; 3rd dose 100 microgramg/kg subcutaneously 21 days after the 2nd dose) resulted in significant upregulation of perforin at all doses in CD4 and CD8 T cells in peripheral blood sampled on day 3 post dosing in comparison to 3 SIV+ control animals. Interestingly, perforin increase was noted in all CD4 and CD8 T cell subsets (naive, central memory, effector memory) and fold increase was most prominent in naïve CD4 cells $(2.7$ fold, $\mathrm{P}=0.05)$ and effector memory CD8 T cells ( 2 fold, $\mathrm{P}=0.0004$ ). Perforin upregulation was accompanied by slight increase in Ki67 in CD8 T cells, but with no evidence of cellular expansion and significant decrease in HLA-DR expression in naïve and central memory CD4 T cells. No change in CD4 counts or virus load were noted. Functional virus specific $\mathrm{T}$ cell responses and serum anti-IL-21 determination are ongoing.

\section{Conclusion}

The novel perforin inducing property of IL-21 in CD4 and CD8 T cells without inducing CD4 T cell activation makes it an attractive molecule for consideration in immunotherapeutic and vaccine strategies. 